\title{
Violência contra pessoas idosas: um olhar sobre o fenómeno em Portugal $^{1}$
}

\author{
Maria Isabel Dias \\ Universidade do Porto, Porto, Portugal \\ mdias@letras.up.pt \\ Alexandra Lopes \\ Universidade do Porto, Porto, Portugal \\ aslopes@letras.up.pt \\ Rute Lemos \\ Universidade do Porto, Porto, Portugal \\ rlemos@letras.up.pt
}

\begin{abstract}
Resumo: No presente artigo parte-se do reconhecimento do abuso de idosos como um fenómeno complexo e multidimensional. Dá-se conta da diversidade de molduras teóricas e de evidência empírica que tem marcado a análise deste fenómeno em Portugal, diversidade que se acredita explica algumas das inconsistências que continuam a marcar as tentativas de avaliação da extensão do mesmo. O artigo baseia-se numa revisão sistemática da literatura quer ao nível teórico, quer ao nível dos estudos empíricos realizados em Portugal.
\end{abstract}

Palavras-chave: Abuso de idosos; fatores de risco; estudos de prevalência.

Abstract: This article starts by recognizing elder abuse as a complex and multidimensional phenomenon. It showcases the diversity of theoretical frameworks and empirical evidence that have marked the analysis of this phenomenon in Portugal, a diversity which is believed to explain some of the inconsistencies that continue be present on the attempts of evaluating its' extent. The article is based on a systematic review of the literature both at a theoretical level, as well as at the level of the empirical studies carried out in Portugal.

Keywords: elder abuse; risk factors; prevalence studies.

\footnotetext{
${ }^{1}$ Este artigo enquadra-se no âmbito do projeto "O abuso de idosos: determinantes sociais, económicas e de saúde”, financiado por fundos nacionais através da FCT - Fundação para a Ciência e a Tecnologia, I.P., na sua componente Orçamento de Estado (FCT PTDC/IVC-SOC/6782/2014), e pelo orçamento do programa COMPETE - Programa Operacional e Competitividade na sua componente FEDER (POCI-01-0145FEDER-016670).
} 


\section{Introdução}

O reconhecimento de que os idosos são vítimas de violência na família e em contexto institucional foi tardio. Só no final dos anos 1970, início dos anos 1980, é que foi reconhecido como um grave problema social, ao ponto de recentemente ser considerado, de igual modo, como um problema de saúde pública e uma violação de direitos humanos (Dias, Lopes \& Lemos, 2019; Santos et al., 2018). Inicialmente, foram os profissionais ligados ao serviço social e à saúde que sinalizaram este tema, passando a intervenção a desenvolver-se sobretudo no âmbito das instituições médicas e sociais. Nos últimos anos o fenómeno ganhou um interesse crescente, centrando-se a sua abordagem na definição do conceito, na identificação de tipologias das vítimas e dos agressores, na sinalização de fatores de risco e de proteção dos abusos, no desenvolvimento de teorias explicativas e de modelos de intervenção. Trata-se de um fenómeno multidimensional ao nível das suas manifestações (Santos et al., 2018), pelo que é fundamental a produção de conhecimento científico sobre a diversidade de configurações que o abuso de idosos assume nas nossas sociedades, de forma a sustentar uma intervenção, com qualidade, em todos os contextos socioprofissionais (Santos, 2018).

A produção de conhecimento científico, porém, coloca exigências tanto ao nível concetual como ao nível metodológico e empírico. Por um lado, exige clarificação e consenso em torno dos conceitos, do seu significado e do seu alcance. Por outro lado, exige o desenvolvimento e validação de protocolos metodológicos para observação e medição do fenómeno, condição prévia à sistematização de evidência empírica. Quer um campo, quer o outro têm assistido a desenvolvimentos assinaláveis nas últimas décadas, mas com resultados difusos que, por vezes, dificultam o próprio conhecimento do fenómeno da violência.

Neste artigo procura-se oferecer uma sistematização atualizada dos principais quadros concetuais mobilizados, na literatura, para discussão do fenómeno da violência e abuso de idosos em Portugal, assim como do seu reflexo ao nível da investigação empírica nesse mesmo país

A grande conclusão a que se chega é a de que a fragmentação que persiste na moldura conceptual que é mobilizada para este domínio de análise encontra reflexo na inconsistência de algumas das tendências que se assinalam empiricamente.

\section{Conceito(s) e teorias explicativas do abuso de idosos}

Ainda hoje são inúmeras as divergências concetuais sobre a definição de abuso de idosos, mas foi entre 1975 e 1978 que apareceram na literatura sobre a violência doméstica os termos de "granny bashing", "granny-battering syndrome”, "elder abuse” (Biggs, Phillipson \& Kingston, 1995; Hudson, 1991), entre outros. Os conceitos de "granny bashing”, "granny-battering syndrome" foram usados pela primeira vez por Burston (1975) e Baker (1975) para darem conta da violência exercida sobre as pessoas mais velhas em contexto familiar. São conceitos que estavam muito focados na agressão física e que orientavam a intervenção social e médica para os abusos cometidos em contexto de dependência na velhice (Mysyuk et al., 2013). Progressivamente, com o conceito de "battered elder syndrome" (Block \& Sinnott, 1979), outros tipos de violência começaram a ser alvo de intervenção. Assim, para além da agressão física, o abuso psicológico, financeiro, a par de um conjunto de fatores de risco ligados sobretudo à dependência e ao comportamento dos agressores passaram a estar enquadrados no referido conceito. Ainda 
assim, continuava a ser um conceito que dava mais visibilidade à violência física por força da sua associação ao termo "syndrome” (Mysyuk et al., 2013).

A controvérsia à volta do conceito prosseguiu nas décadas seguintes alargando ora o seu campo semântico, dando visibilidade a outros comportamentos (e.g., abuso emocional, sexual, negligência), ora o leque de vítimas (que não são só as avozinhas) e de agressores (e.g., cuidador formal). Foi esta preocupação que esteve na origem da proposta do conceito de "old age abuse" por Eastman (1984), o qual veio igualmente salientar a natureza sistemática dos abusos.

As décadas de 1990 e de 2000 representam um passo significativo no âmbito da discussão à volta do conceito. Ultrapassa-se assim a definição mais restrita associada ao "granny battering syndrome" e adota-se o conceito de "elder abuse" - abuso de idosos - para dar conta da variedade de omissões, mas também de comportamentos que causam dor e/ou lesões físicas (abuso físico), mal-estar psíquico, emocional e mental (abuso psicológico e emocional), conduzem à apropriação indevida dos recursos, bens ou propriedades do idoso (abuso material ou financeiro), à não satisfação das suas necessidades, sobretudo em situação de dependência (negligência ativa e passiva) e podem, ainda, integrar atividade sexual não consentida (abuso sexual) (Pillemer et al., 2016; Dias, Lopes \& Lemos, 2019). Esta discussão concetual acabou por influenciar a própria definição usada pela OMS (WHO, 2002), referindo-se ao abuso de idosos como sendo qualquer ato isolado ou repetido, ou ausência de uma ação apropriada, que ocorre no âmbito de qualquer relacionamento onde haja uma expectativa de confiança, causador de dano ou incómodo a uma pessoa idosa.

A partir da 2. ${ }^{a}$ Assembleia Mundial sobre o Envelhecimento, várias organizações internacionais (e.g., ONU, OMS, Conselho da Europa) passaram a referir-se a este fenómeno como um comportamento destrutivo, ou à falta de ação apropriada, que ocorre num contexto de uma relação de confiança, no quadro da vida familiar ou institucional, cuja frequência, única ou repetida, causa dano ou sofrimento físico, psicológico e emocional à pessoa idosa, configurando uma séria violação dos direitos humanos (Gil et al., 2012). Para Soares et al. (2010), o conceito designa quaisquer comportamentos abusivos, infligidos sobre pessoas com 60 ou mais anos, que colocam em perigo a sua integridade física, psicológica, emocional, verbal, financeira ou material, prejudicando o seu bem-estar.

Independentemente das variações, é inegável que o conceito em causa integra vários tipos de violência designadamente o abuso físico, psicológico, material, a negligência, a molestação sexual, entre tantos outros tipos de violência. É inesgotável a lista de comportamentos ilustradora da multiplicidade de maus-tratos infligidos aos idosos na família, mas também em instituições, como é o caso dos lares e centros de apoio e acolhimento. Nestas instituições, por vezes, os idosos são vítimas de sub ou sobre medicação, de agressão verbal, negligência, de abuso psicológico e financeiro. Também são alvo de infantilização, despersonalização e de processos de desumanização. Nestes espaços, os idosos tornam-se vítimas particularmente vulneráveis quando o seu estado de dependência e de saúde, em geral, é precário e se encontra isolado da sua rede de parentesco, dos amigos e da comunidade. As pessoas idosas raramente denunciam o abuso institucional porque têm medo de sofrerem retaliações e, na maior parte dos casos, não têm consciência dos seus direitos legais (Dias, 2010). Nestas instituições a violência ocorre devido a fatores macrossociais (e.g., cultura da violência, idadismo, conflitos intergeracionais), institucionais (e.g., falta de condições de trabalho, sobrecarga dos profissionais, ausência de formação profissional, inadequação das infraestruturas) e individuais (e.g., comportamento e personalidade dos cuidadores formais, desgaste físico e mental, questões morais e éticas) (Ribeirinho, 2019). 
Apesar dos desenvolvimentos supramencionados e do alargamento do tipo de comportamento que a noção de abuso de idosos integra, ainda persistem questões críticas no plano concetual, com implicações sérias na medição do fenómeno e na comparação dos resultados (Santos, 2018). Em vários estudos continua a ser comum o uso de diferentes terminologias e outras formas de abuso (e.g., autoabuso ao autonegligência), alguma pesquisa ainda é descritiva e com um pendor pragmático, ou seja, não apresenta grandes desenvolvimentos concetuais, nem resultados práticos. Ora estas dificuldades refletem-se quer no âmbito da investigação científica, quer ao nível da definição de modelos de intervenção no fenómeno. Importa, então, ter em linha de conta que o abuso de idosos constitui um fenómeno multidimensional estando, por isso, associado a um conjunto de fatores de risco relacionados com as características das vítimas, dos agressores e do contexto social (Gil et al, 2015).

\section{Os fatores de risco: a ausência de teorias explicativas integradas}

A ausência de consenso acerca da definição de abuso de idosos, reflete, igualmente, a dificuldade em desenvolver um quadro teórico integrado. Esta dificuldade também decorre da assunção de que o abuso é um fenómeno multifacetado dificilmente explicável por uma teoria unificada (Santos, 2018). Por esta razão, a pesquisa tem-se focado na identificação dos fatores de risco, mais do que no desenvolvimento de teorias explicativas integradas. Neste sentido, Wolf e Pillemer (1989) referem quatro perspetivas teóricas que identificam os fatores de risco e propõem a explicação dos abusos, nomeadamente: as dinâmicas intra-individuais; a transmissão intergeracional do comportamento violento; o stress dos cuidadores e a perspetiva do isolamento social. A teoria das dinâmicas intraindividuais considera que os idosos que estejam sob os cuidados de familiares que manifestem problemas mentais, emocionais ou outros traços psicopatológicos correm um risco superior de serem vítimas de abusos. O mesmo sucede quando há ocorrência de comportamentos aditivos. A transmissão intergeracional do comportamento violento argumenta que a exposição e a experiência de violência durante a infância conduzem à aprendizagem de comportamentos abusivos e provavelmente à sua reprodução. Postula que as pessoas que perpetram abusos sobre os idosos foram educadas em contextos familiares violentos. O stress experimentado pelos indivíduos no exterior da família é igualmente apontado como um fator de risco de abusos sobre os idosos. Acontecimentos como o desemprego, dificuldades financeiras, podem potenciar nos indivíduos comportamentos violentos. O isolamento social é igualmente considerado como um fator de risco para os idosos, pelo que as redes sociais de apoio são importantes, quer como fator de moderação do stress e das tensões experimentadas pelas famílias que têm idosos a seu cargo, quer como meio de combater o referido isolamento social.

Um argumento caro à pesquisa gerontológica tem a ver com os níveis de dependência dos idosos face aos cuidados dos membros da família (sobretudo cônjuges e filhos/as), mas também com a dependência destes em relação aos idosos, seja no plano financeiro, seja ao nível da coresidência, situação esta que é considerada por muitos investigadores como um fator de risco importante (Wolf \& Pillemer, 1989). Por seu turno, o modelo sociológico acrescenta aos fatores de risco supramencionados os seguintes: a incapacidade funcional e mental da pessoa idosa; problemas de saúde mental no cuidador; problemas económicos; comportamentos aditivos do agressor (e.g., alcoolismo, toxicodependência, vício do jogo); situações de isolamento, pobreza e exclusão social, entre outros fatores (Dias, Lopes \& Lemos, 2019; Ribeirinho, 2019).

Já o modelo socioecológico, utilizado pela OMS, vê o abuso de idosos como uma complexa teia de relações entre três níveis analíticos ou subsistemas distintos, 
nomeadamente: o nível macro (e.g., determinantes estruturais e sistema de crenças, normas e atitudes face à pessoa idosa); exo e meso (e.g., sistema de relações de trabalho, redes sociais de suporte formal e informal) e o nível micro (e.g., tipo de relações familiares conjugais, filiais, contexto familiar, isolamento social) (Gil et al., 2014; Dias, Lopes \& Lemos, 2019).

Nenhuma destas teorias obteve acordo suficiente para serem usadas como um quadro teórico preferencial na explicação dos abusos. A única certeza neste campo é que devido às múltiplas configurações dos abusos ao nível das vítimas, dos agressores (familiares, cuidadores formais, amigos, etc.) e dos contextos de ocorrência (família/instituições) nenhuma teoria, por si só, é suficiente para dar conta do fenómeno (Santos, 2018).

Independentemente da diversidade dos fatores de risco explicativos, do fato de a violência ser cometida em contexto familiar ou institucional, o seu efeito é muito nefasto causando nos idosos danos físicos, psicológicos, emocionais e morais. Os idosos vítimas sentemse geralmente culpados dos abusos que lhes são infligidos, desenvolvem atitudes de isolamento, baixa autoestima e depressão. A presença de profissionais do serviço social, da saúde, das forças de segurança pública, entre outros, torna mais difícil a prática de abusos sobre esta população. O apoio comunitário, a integração na rede alargada das relações de parentesco, amicais e de vizinhança são medidas importantes inibidoras da ocorrência de abusos sobre os mais velhos na família e em instituições.

\section{Estudos de prevalência: da fragmentação teórica à fragmentação empírica}

A partir da década de 1990, e em particular no meio académico, assiste-se a um crescente interesse pelo conhecimento da extensão do abuso de idosos com a realização de estudos de prevalência baseados em amostras representativas da população. No entanto, observam-se variações nos resultados da prevalência estimada pelo facto de se diferenciarem relativamente ao enquadramento concetual do abuso, aos instrumentos de medição de situações abusivas, às referências temporais, entre outros pressupostos teóricos e metodológicos (Santos, Nicolau, Fernandes \& Gil, 2013; Lindert et al., 2012). Apesar da comparabilidade dos dados por vezes ficar comprometida, estes estudos são importantes quer para projeção do fenómeno como um grave problema social e de saúde pública, quer por permitirem uma estimação aproximada da sua ocorrência.

Para identificar os estudos de prevalência realizados em Portugal, procedeu-se a uma revisão sistemática da literatura nacional sobre a problemática através da consulta às bases que integram a base EBSCOHost, repositórios científicos portugueses, revistas científicas nacionais e pesquisa em sites de centros de investigação em domínios científicos nos quais a temática se enquadra (Ciências Sociais e Humanas, Ciências da Saúde e Ciências Jurídicas) ${ }^{2}$. Os critérios de inclusão dos estudos de prevalência de abuso de idosos foram os seguintes: recurso a amostras representativas da população; ter a delimitação territorial do estudo explicitada, de âmbito nacional ou regional; reportar-se a pessoas com 60 ou mais anos. No total foram selecionados apenas cinco estudos, dois dos quais de âmbito regional, - um na região de Évora (Lopes et al., 2012) outro na Amadora (Pires, 2009) - dois de âmbito europeu - o projeto ABUEL (Soares et al., 2010) e o projeto AVOW (Luoma et al., 2011), este último com a singularidade de abordar exclusivamente a violência infligida contra mulheres idosas- e apenas um de alcance

\footnotetext{
${ }^{2} \mathrm{O}$ protocolo da pesquisa de estudos de prevalência pode ser consultado mediante solicitação do mesmo às autoras do artigo.
} 
nacional (Gil et al, 2014). Para efeitos da análise excluiu-se o de Pires (2009), uma vez que os dados se reportavam à vivência de situações de violência ao longo da vida, enquanto os restantes focavam os últimos 12 meses.

As pesquisas empíricas selecionadas apresentam critérios similares de recrutamento dos participantes, nomeadamente todos têm a capacidade cognitiva intacta, residem em domicílios particulares e têm idade superior a 60 anos, com exceção do estudo de Lopes et al (2012) cuja idade mínima era 65 anos.

A tabela 1 sintetiza a quantificação estimada de abuso de idosos em Portugal segundo os estudos mencionados. 
Tabela 1. Caracterização de estudos de prevalência de abuso de idosos em Portugal e resultados de prevalência segundo a natureza dos abusos e sexo das vítimas (\%)

\begin{tabular}{|c|c|c|c|c|c|}
\hline & $\begin{array}{l}\text { Autor(es), } \\
\text { ano }\end{array}$ & Soares et al., 2010 & Luoma et al., 2011 & $\begin{array}{l}\text { Gil et al., } \\
2014\end{array}$ & Lopes et al., 2012 \\
\hline & Âmbito & $\begin{array}{c}\text { Transnacional } \\
\text { europeu }\end{array}$ & $\begin{array}{c}\text { Transnacional } \\
\text { europeu }\end{array}$ & Nacional & Regional: Évora \\
\hline & opulação-alvo & $\begin{array}{l}\text { Indivíduos entre } 60 \\
\text { e } 84 \text { anos, a residir } \\
\text { em domicílios } \\
\text { particulares }\end{array}$ & $\begin{array}{c}\text { Mulheres com } 60 \\
\text { ou mais anos, a } \\
\text { residir em } \\
\text { domicílios } \\
\text { particulares }\end{array}$ & $\begin{array}{c}\text { Indivíduos com } 60 \\
\text { e mais ano, a } \\
\text { residir em } \\
\text { domicílios } \\
\text { particulares }\end{array}$ & $\begin{array}{c}\text { Indivíduos com } 65 \\
\text { ou mais anos }\end{array}$ \\
\hline Dir & ensão da amostra & $\mathrm{n}=656$ (Portugal) & $\mathrm{n}=649$ (Portugal) & $n=1123$ & $\mathrm{n}=903$ \\
\hline Instr & $\begin{array}{l}\text { Imentos de recolha } \\
\text { de dados }\end{array}$ & $\begin{array}{c}\text { Adaptação CTS2 e } \\
\text { O'Keeffle }\end{array}$ & $\begin{array}{l}\text { Adaptação CTS2 e } \\
\text { questões próprias }\end{array}$ & Questões próprias & $\begin{array}{c}\text { Adaptação CTS2 e } \\
\text { O’Keeffle }\end{array}$ \\
\hline & $\begin{array}{l}\text { odo de recolha de } \\
\text { dados }\end{array}$ & $\begin{array}{c}\text { Inquérito } \\
\text { presencial }\end{array}$ & Inquérito via postal & $\begin{array}{l}\text { Inquérito por } \\
\text { telefone }\end{array}$ & $\begin{array}{l}\text { Inquérito } \\
\text { presencial }\end{array}$ \\
\hline & odo de referência & Últimos 12 meses & Últimos 12 meses & Últimos 12 meses & Últimos 12 meses \\
\hline 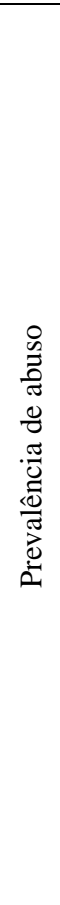 & \begin{tabular}{|l} 
Psicológico \\
Total \\
Homens \\
Mulheres \\
Financeiro \\
Total \\
Homens \\
Mulheres \\
Sexual \\
Total \\
Homens \\
Mulheres \\
Físico \\
Total \\
Homens \\
Mulheres \\
Lesões físicas \\
Total \\
Homens \\
Mulheres \\
Negligência \\
Total \\
Homens \\
Mulheres
\end{tabular} & $\begin{array}{l}21,9 \\
16,6 \\
25,4 \\
\\
7,8 \\
9,6 \\
6,6 \\
\\
1,3 \\
0,8 \\
1,6 \\
\\
2,1 \\
2,2 \\
2,0 \\
\\
0,7 \\
0,0 \\
1,2\end{array}$ & $\begin{array}{c}- \\
- \\
32,9 \\
- \\
- \\
16,5 \\
- \\
- \\
3,6 \\
- \\
- \\
2,8 \\
- \\
- \\
- \\
- \\
- \\
9,9\end{array}$ & $\begin{array}{c}6,3 \\
5,3 \\
7,0 \\
0,2 \\
- \\
- \\
2,3 \\
- \\
- \\
- \\
- \\
- \\
0,4 \\
- \\
-\end{array}$ & $\begin{array}{c}30,0 \\
- \\
- \\
7,0 \\
- \\
- \\
2,0 \\
- \\
- \\
4, \\
4 \\
- \\
- \\
1,0 \\
- \\
- \\
- \\
- \\
-\end{array}$ \\
\hline
\end{tabular}

Os de âmbito transnacional europeu (Soares et. Al, 2010 e Luoma et al., 2011) bem como o regional (Lopes et al., 2012) apresentam uma maior proporção de pessoas idosas vítimas de violência comparativamente com o estudo nacional. Por sua vez, o projeto AVOW (Luoma et al., 2011) detém a prevalência mais elevada para todos os tipos de abuso, facto que deverá ser considerado tendo em linha de conta que a unidade de análise foi exclusivamente mulheres.

O tipo de abuso mais reportado, para o total dos participantes independentemente do sexo, é o psicológico, com valores entre os $30,0 \%$ e os $6,3 \%$, seguido pelo financeiro, cujos resultados oscilam entre 7,8\% e 6,3\%. Porém, no estudo de âmbito nacional (Gil et al., 2014), o abuso psicológico e o financeiro assumem a mesma proporção de idosos agredidos.

Relativamente à distribuição da prevalência de abuso segundo o sexo das vítimas, os resultados dos estudos estão alinhados com o descrito na literatura constatando-se, na generalidade, evidências empíricas de assimetrias de género no fenómeno em análise na medida em que as mulheres são predominantes entre as vítimas de violência. Contudo, se no estudo de Gil et al. (2014) as mulheres reportam ter vivenciado mais abuso 
psicológico e financeiro, não tendo sido divulgados dados por sexo para os outros tipos de abuso, no estudo de Soares et al., 2010, as mulheres apresentam prevalência superior na violência psicológica, sexual, com lesões físicas bem como negligência e, por contraposição, são os homens que estão mais expostos ao risco de abuso financeiro e físico, ainda que neste último a diferença entre homens e mulheres tenha sido apenas de uma décima.

Para uma análise mais detalhada dos contextos de violência, os estudos de Soares et al. (2011) e Gil et al. (2014) abordaram igualmente o tipo de relação que as vítimas mantinham com os agressores. Em ambos os estudos os homens são assinalados como principais agressores. No caso das vítimas mulheres, os agressores são predominantemente indivíduos da esfera da intimidade atual ou passada, seguido pelos descendentes masculinos. Por sua vez entre as vítimas do sexo masculino verifica-se situação inversa (Gil et al., 2014).

Paralelamente aos estudos de prevalência, a análise de dados de fontes oficiais relativos a denúncias contribui para o conhecimento do quantitativo de ocorrência de situações abusivas em idosos. Em Portugal destacam-se em particular duas fontes de dados estatísticos, designadamente as forças de segurança (Polícia de Segurança Pública - PSP - e Guarda Nacional Republicana - GNR) e a Associação Portuguesa de Apoio à Vítima (APAV), uma organização não-governamental. As opções de tratamento e divulgação dos dados revelam que ambas as instituições privilegiam o enquadramento concetual de abuso de idosos como subsidiário do conceito mais amplo de violência doméstica. Destaca-se, ainda, que assumem o mesmo critério de definição dos limites etários para classificação de uma pessoa como idosa, designadamente ter 65 ou mais anos.

De acordo com dados das autoridades policiais, a proporção de idosos vítimas violência doméstica aumentou de 6,9\% em 2010 para 9,0\% em 2016 (DGAI, 2011, SGMAI, 2017). Como se pode observar na tabela 2, o efeito de género é marcante na distribuição de vítimas de violência doméstica em todos os escalões etários. Contudo, e considerando apenas a população adulta, é nos escalões etários dos 65 ou mais anos que a proporção de mulheres vítimas é inferior, pelo que se pode concluir que para os homens a idade constitui um fator de vulnerabilidade de risco de exposição a situações de violência.

Tabela 2 - Taxa de feminização das vítimas de violência doméstica por escalões etários (dados relativos ao ano de 2014)

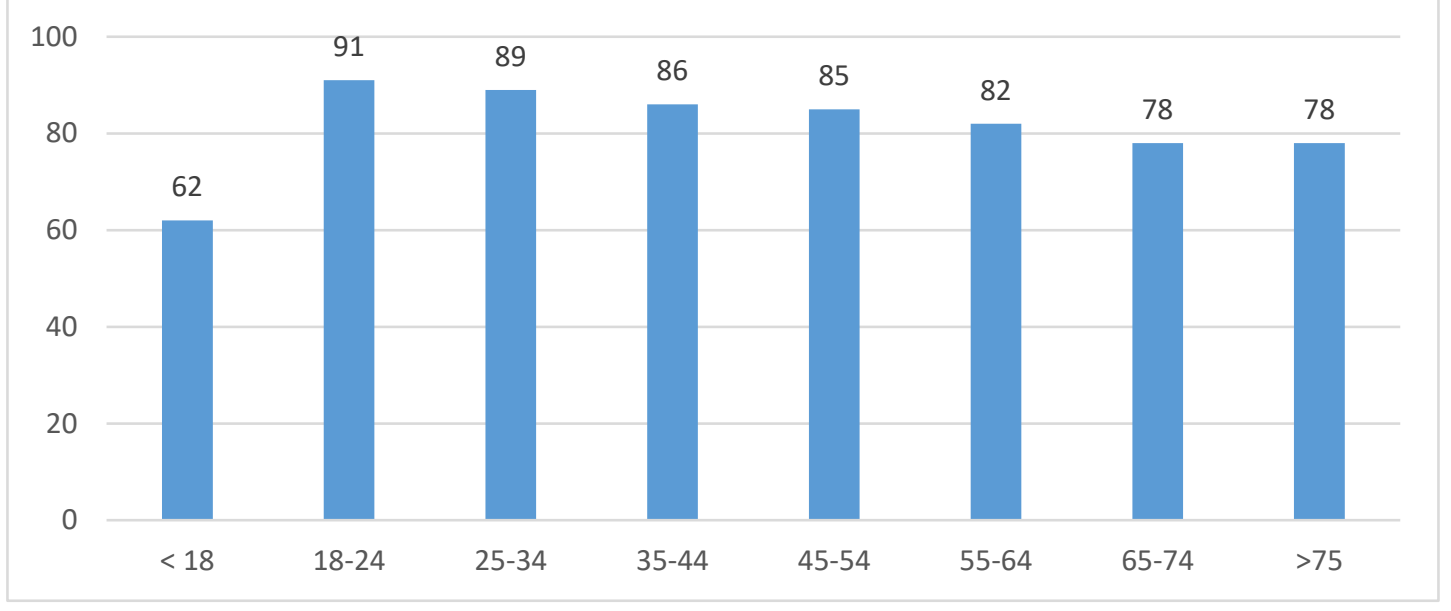

Fonte: SGMAI, 2015.

Os dados das autoridades de segurança revelam ainda a existência de um padrão no que concerne aos laços sociais entre a vítima e o agressor segundo os grupos etários das vítimas e que não varia de acordo com sexo das mesmas. Para as que têm entre 65 e 74 
anos, o agressor enquadra-se na maioria das situações no contexto de relação de intimidade/conjugalidade, ao passo que entre os que têm 75 ou mais anos, os ascendentes apresentam-se como os principais agressores (SGMAI, 2015) ${ }^{3}$.

De igual modo, os dados estatísticos disponibilizados pela APAV revelam um aumento no número de idosos que reportam vivenciar situações de violência doméstica àquela instituição, na ordem dos 17\%, entre 2010 (n=608) e 2017 (n=711) (APAV 2014, 2018). Os maus tratos psíquicos (38,3\%) e maus tratos físicos $(25,6 \%)$ constituem os tipos de crime de violência doméstica mais denunciados à APAV. Para 48\% dos idosos vítimas de violência doméstica os agressores são os filhos (APAV, 2018).

No entanto, são reconhecidas as limitações na leitura dos dados oficiais uma vez que grande parte dos casos de abuso não são detetados pelos serviços oficiais nem são reportados, quer pelas próprias vítimas, quer por familiares ou profissionais que acompanham os idosos (Berman \& Lachs, 2011; Dong, 2015). A decisão dos idosos em reportar a situação de vivência de abuso está associada a diversos fatores como a negação, medo de retaliação ou abandono por parte do agressor, vergonha e não ter conhecimento onde se dirigir para procurar apoio (Mysyuk, Westendorp \& Lindenberg, 2016; Begley, O’Brien, Anand, Killick \& Taylor, 2012). De igual modo, o facto de os idosos assumirem diferentes entendimentos das situações que podem ser consideradas como abusivas, alguns atos de violência por eles vivenciados podem não ser reconhecidos como tal (Idem).

\section{Conclusão}

A análise aos dados de estudos de prevalência, bem como das fontes oficiais sugere que, em Portugal, a violência contra idosos está a aumentar, a aumentar de facto e a aumentar quando medida a partir da que é reportada. A primeira tende a ser, porém, superior à segunda. As vítimas de violência contra idosos são na sua maioria mulheres, muito embora a percentagem de homens esteja a crescer. Quanto aos agressores, são na sua maioria do sexo masculino, independentemente do sexo da vítima, o que poderá estar associado a um padrão normativo de dominância masculina tão característico da sociedade portuguesa. Poderá ainda ser indiciador da reprodução do ciclo de violência em que muitas mulheres estão envolvidas desde a juventude e que se prolonga até à idade avançada. Os traços empíricos identificados, sugerem, assim, a permanência de inconsistências nas estimativas e de imprecisões na forma como o fenómeno é percebido e medido.

Apesar da fragmentação teórica e empírica que caracteriza esta área é inegável que a violência contra pessoas idosas é um constructo que engloba fenómenos distintos (violência nas relações íntimas, negligência, etc.) mas que, não obstante, partilham características comuns, sobretudo ao nível dos efeitos nas vítimas. É também um fenómeno que nos obriga a considerar na sua análise diferentes dimensões como, por exemplo, a díade vítima-agressor, os tipos de comportamentos abusivos, o seu grau de severidade e frequência, os fatores de risco, assim como os diferentes contextos de ocorrência, com vista a darmos conta das dinâmicas e das diversas configurações que o abuso de idosos assume nas nossas sociedades (Santos, 2018).

\footnotetext{
${ }^{3}$ No caso de vítimas do sexo feminino, no escalão etário dos 65-74 anos, $65 \%$ dos agressores são cônjuges ou ex-cônjuges e $32 \%$ descendentes da vítima; no escalão etário dos 75 ou mais anos $60 \%$ dos agressores são descendentes e $38 \%$ cônjuges ou ex-cônjuges. No caso de vítimas do sexo masculino, no escalão etário dos 65-74 anos, 56\% dos agressores são cônjuges ou ex-cônjuges e 41\% descendentes da vítima; no escalão etário dos 75 ou mais anos $67 \%$ dos agressores são descendentes e $29 \%$ cônjuges ou ex-cônjuges.
} 
Apesar das dificuldades apontadas, todos os estudos têm contribuído para um melhor conhecimento ao nível da caracterização geral do fenómeno, dos agressores e dos perfis das vítimas mais vulneráveis, que são sobretudo os idosos mais velhos (mais de 75 anos), do género feminino, mais dependentes, com recursos escassos do ponto de vista das condições socioeconómicas, e os mais desprotegidos ao nível das redes médico-sociais, familiares e comunitárias (Dias, Lopes \& Lemos, 2019; Dias, 2005; Ribeirinho, 2019).

Não obstante, esta é uma área que tem ainda muito por resolver, nomeadamente ao nível da adoção de uma terminologia e de modelos teóricos que sejam minimamente consensuais. A variabilidade de conceitos, definições e concetualizações afeta, nem sempre positivamente, a validade da evidência, da medida do fenómeno e das políticas sociais implementadas com base nos seus resultados (Santos, 2018). Por isso, neste campo, não só é urgente a promoção de uma abordagem multidimensional do problema, como uma maior consistência teórica e empírica sobre o abuso de idosos.

\section{Bibliografia}

Associação Portuguesa de Apoio à Vítima, APAV . (2014). Crimes de violência doméstica [2000-2012].

https://apav.pt/apav_v3/index.php/pt/estatisticas-apav

Associação Portuguesa de Apoio à Vítima, APAV. (2018). Crimes de Violência Doméstica - Violência Filioparental 2013-2017. Disponível em: https://apav.pt/apav_v3/index.php/pt/estatisticas-apav

Baker, A.A. (1975). Granny-battering. Modern Geriatrics, 5, 20-24.

Begley, E., O'Brien, M., Anand, J. C., Killick, C., \& Taylor, B. (2012). Older people's views of support services in response to elder abuse in communities across Ireland. Quality in Ageing and Older Adults, 13(1), 48-59. doi:10.1108/14717791211213616

Berman, J., \& Lachs, M. Under the Radar: New York State Elder Abuse Prevalence Study. New York: Lifespan of Greater Rochester. 2011. Disponível em: http://ocfs.ny.gov/main/reports/Under\%20the\%20Radar\%2005\%2012\%2011\%20final

\%20report.pdf Acedido em: 19 Maio 2017.

Block, M., \& Sinnott, I. (1979). The Battered Elder Syndrome. University of Maryland Press, Maryland.

Burston, G.R. (1975). Granny battering. British Medical Journal, 3, 592.

Carvalho, M. I. (2019). Percursos Legislativos e Interventivos no combate à violência. In: Paulino, M. \& Costa, M. (coords.): Maus-Tratos a Pessoas Idosas. Lisboa: Pactor.

Dias, I. (2005). Envelhecimento e violência contra os idosos. Sociologia, 15, pp. 249-273. Dias, I. (2010). Violência na Família. Uma abordagem Sociológica. Porto: Edições

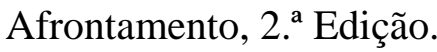

Dias, I., Lopes, A., \& Lemos, R. (2019). O Abuso de Pessoas Idosas: Definições e Controvérsias. In: Paulino, M. \& Costa, M. (coords.): Maus-Tratos a Pessoas Idosas. Lisboa: Pactor.

Direção Geral da Administração Interna, DGAI (2011). Violência Doméstica 2010. Ocorrências participadas às forças de Segurança. Disponível em: https://www.dgai.mai.gov.pt/files/conteudos/VD_Relatorio\%20Anual_2010_2_5_2011. pdf

Dong, X. Q. (2015). Elder Abuse: Systematic Review and Implications for Practice. J Am Geriatr Soc, 63(6), 1214-1238. doi:10.1111/jgs.13454

Eastman, M. (1984). Old Age Abuse. Age Concern England.

Gil, A. P., Faria, P. L., Gomes, I., \& Fonseca R. (2012). Perspetivas atuais sobre a proteção jurídica da pessoa idosa vítima de violência familiar: contributo para uma 
investigação em saúde pública. Revista Portuguesa de Saúde Pública, 30 (2), pp. 149162.

Gil, Ana Paula (Ed. Responsável). Projeto Envelhecimento e Violência. Instituto Nacional de Saúde Dr. Ricardo Jorge, IP, Departamento de Epidemiologia, Lisboa. 2014. Disponível em: http://www.insa.min-saude.pt/publicacao-envelhecimento-e-violencia/ Acedido em: 14 de março de 2019.

Gil, A. P. et al. (2015). Elder abuse in Portugal: Findings from the first national prevalence study. Journal of Elder Abuse and Neglect, 27, pp. 174-195.

Hudson, M.F. (1991). Elder mistreatment: a taxonomy with definitions by Delphi. Journal of Elder Abuse and Neglect, 3. pp. 1-20.

Lindert, J., Luna, J., Torres-Gonzalez, F., Barros, H., Ioannidi-Kapolou, E., Quattrini, S., ... Soares, J. J. (2012). Study design, sampling and assessment methods of the European study 'abuse of the elderly in the European region'. Eur J Public Health, 22(5), 662-666. doi:10.1093/eurpub/ckr079

Lopes, M., Escoval, A., Mendes, F., Pereira, D., Pereira, C., Carvalho, P. \& Fonseca, C. (2012). Violência, abuso, negligência e condição de saúde dos idosos: Relatório Final. Direção Geral da Saúde, Universidade de Évora, Universidade Nova de Lisboa, Escola Nacional de Saúde Pública.

Luoma, M.L., Koivusilta, M, Lang, G., Enzenhofer, E., De Donder, L., Verté, D. \& Penhale, B. (2011). Prevalence Study of Abuse and Violence against Older Women. Results of a Multi-cultural Survey Conducted in Austria, Belgium, Finland, Lithuania, and Portugal (European Report of the AVOW Project). Finland: National Institute for Health and Welfare (THL).

Marshall, V. W (1999). Analysing social theories of aging. In: Bengtson, Vern L. \& Schaie, K. Warner (Eds.). Handbook of Theories of Aging, New York: Springer Publishing Company. pp. 434-455.

Mysyuk, Y., Westendorp, R. G., \& Lindenberg, J. (2013). Added value of elder abuse definitions: a review. Ageing Res Rev, 12(1), 50-57. doi:10.1016/j.arr.2012.04.001

Mysyuk, Y., Westendorp, R. G. J., \& Lindenberg, J. (2016). Older persons' definitions and explanations of elder abuse in the Netherlands. Journal of Elder Abuse and Neglect, 28(2), 95-113. doi:10.1080/08946566.2015.1136580

Pillemer, K., Burnes, D., Riffin, C., \& Lachs, M. S. (2016). Elder Abuse: Global Situation, Risk Factors, and Prevention Strategies. Gerontologist, 56 Suppl 2, S194-205. doi:10.1093/geront/gnw004

Pires, S. (2009). Violência Sobre Idosos. Amadora, Câmara Municipal da Amadora.

Ribeirinho, C. (2019). Violência contra pessoas idosas em contexto institucional: (re)conhecimento e intervenção. In: Paulino, M. \& Costa, M. (coords.): Maus-Tratos a Pessoas Idosas. Lisboa: Pactor.

Santos, A. J., Nicolau, R., Fernandes, A. A. \& Gil, A. P. (2013). Prevalência da violência contra as pessoas idosas: uma revisão crítica da literatura. Sociologia: Problemas $e$ Práticas, 72, 53-77.

Santos, A. J.; Nunes, B.; Kislaya, I., Gil, A. P.; Ribeiro, O. (2018). Older adults' emotional reactions to elder victimisation determinants. Health and Social Care in the community, pp. 1-12.

Santos, A. J. (2018). Violence against older adults: multidimensional perspective. Tese de Doutoramento do Programa doutoral em Geriatria e Gerontologia. Universidade do Porto.

Secretaria-geral do Ministério da Administração Interna, SGMAI. (2015). Violência Doméstica 2014. Relatório anual de monitorização. Disponível em: 
https://www.sg.mai.gov.pt/Noticias/Paginas/Viol\%C3\%AAncia-dom\%C3\%A9stica2014---Relat\%C3\%B3rio-anual-de-monitoriza\%C3\%A7\%C3\%A3o-.aspx Acedido a 1 de Março de 2019.

Secretaria-geral do Ministério da Administração Interna, SGMAI. (2016). Violência Doméstica 2015. Relatório anual de monitorização. Disponível em: https://www.sg.mai.gov.pt/Noticias/Paginas/Viol\%C3\%AAncia-dom\%C3\%A9stica2015---Relat\%C3\%B3rio-anual-de-monitoriza\%C3\%A7\%C3\%A3o.aspx Acedido a 1 de Março de 2019.

Secretaria-geral do Ministério da Administração Interna, SGMAI. (2017). Violência Doméstica 2016. Relatório anual de monitorização. Disponível em: https://www.sg.mai.gov.pt/Documents/Rel\%20VD\%202016_v22dez2017vfinal.pdf Acedido a 1 de Março de 2019.

Soares, J., Barros, H., Torres-Gonzales, F., Ionnidi-Kapolou, E., Lamura, G., Lindert, J. \& Stankunas, M. (2010). Abuse and health among eldery in Europe. Kaunas: Lithuanian University of Health Sciences Press.

Wolf, R. S.; Pillemer, K. A. (1989). Helping Elderly Victims - The Reality of Elder Abuse, New York: Columbia University Press.

World Health Organization (WHO) (2002). The Toronto Declaration on the Global Prevention of Elder Abuse. Geneva: World Health Organization. 